\title{
ON THE EXISTENCE OF INVARIANT MEASURES THAT BEHAVE LIKE AREA
}

\author{
STEWART BALDWIN
}

(Communicated by Kenneth B. Meyer)

\begin{abstract}
A countably additive measure on a locally compact, separable, metric space $X$ will be called area-like if points have measure zero, compact sets have finite measure, and nonempty open sets have nonzero measure. Given a homeomorphism $f$ of $X$, we examine criteria that guarantee the existence or nonexistence of arealike measures that are invariant under $f$.
\end{abstract}

In a list of questions circulated after the 1986 Spring Topology Conference, Morton Brown asked the question "When does a Brouwer homeomorphism preserve some nice measure?" In [BS] a partial answer to this question was given by the following result.

Theorem (Baldwin-Slaminka). Suppose $f$ is an orientation preserving homeomorphism of the plane having no fixed points. Then there is a measure on the plane, invariant with respect to $f$, that gives points measure zero, nonempty open sets positive measure, and bounded sets finite measure.

By a well-known theorem of Oxtoby and Ulam [OU], given any Borel measure $\mu$ on the plane that is zero on points, positive on nonempty open sets, finite on bounded sets, such that $\mu\left(R^{2}\right)$ is infinite, there is an autohomeomorphism $h$ of $R^{2}$ such that $\mu(h(B))$ is just the Lebesgue measure of $B$, for any Borel set $B$. If $\mu\left(R^{2}\right)$ is finite, then the same result holds for some homeomorphism between $R^{2}$ and the open disc of the appropriate radius. Thus, the conclusion of the above theorem is equivalent to saying that $f$ is conjugate to some area-preserving homeomorphism (either on the plane or the open unit disk, depending on whether the total measure is finite or infinite). In [BS] an example was given to show that it may not be possible to get the measure in question to be absolutely continuous with respect to Lebesgue measure, even in the fixed point free case. The extent to which this result answers Brown's question depends on the definition of the term "nice." The property we wish to examine in this paper could be loosly stated as "behaves like area." Although the primary motivation here comes from maps on the plane, it will be useful to isolate the properties of area that we need in order to get results on more general spaces.

Received by the editors July 30, 1990.

1991 Mathematics Subject Classification. Primary 28D05, 58F11; Secondary 54H20.

Key words and phrases. Invariant measure, area-preserving homeomorphism. 
Definition. Let $X$ be a locally compact, separable metric space, and let $\mu$ be a countably additive Borel measure on $X$. Then we will say that $\mu$ is arealike iff $\mu(x)=0$ for all $x \in X, \mu(U)>0$ for all nonempty open subsets $U$ of $X$, and $\mu(K)$ is finite for all compact subsets $K$ of $X$. A measure $\mu$ such that $\mu(x)=0$ for all $x \in X$ is generally called atomless. If $f$ is a continuous function on $X$, then $\mu$ is said to be invariant with respect to $f$ if $\mu(B)=\mu\left(f^{-1}(B)\right)$ for every Borel set $B$. If $f$ is a homeomorphism of $X$, then $f^{-1}(B)$ can be replaced by $f(B)$.

The basic problem that we want to examine in this paper is to look for criteria that would guarantee the existence of an arealike invariant measure, given a fixed homeomorphism (or, if possible, just a continuous function). One basic result on the construction of invariant measures is the following classical theorem.

Theorem (Kryloff-Bogoliouboff). Let $X$ be a compact metric space, and let $f$ be a continuous function on $X$. Then there is a Borel measure on $X$ that is invariant with respect to $f$. See [KB]. For a proof in English, [W] is a good reference.

Unfortunately, the above theorem is difficult to use directly for our purposes, since there is no way of telling what the measure looks like. There are many examples in which the invariant measure must give some points nonzero measure. It is necessary to take a closer look at how certain sets acquire their measure. It is interesting to note that the argument given in Theorem 2 can be used to give an alternate proof of the Kryloff-Bogoliouboff Theorem, which is more topological in nature, rather than proceeding through the more classical route via the Riesz Representation Theorem.

We would first like to exclude some trivial, uninteresting cases. Clearly, no space with isolated points can have an arealike measure, and the same is true of any nonempty countable space. Other pathologies (such as the lack of a countable basis) present problems in the proofs that we will not consider here. Thus, in this paper we will examine only nonempty locally compact, separable metric spaces having no isolated points. In order to shorten the statements of the definitions and theorems, we will denote the class of all such spaces by $\Gamma$.

Definition. If $X \in \Gamma$, let $H(X)$ be the set of all autohomeomorphisms of $X$. Given $X \in \Gamma$ and $f \in H(X)$, the wandering set of $f$, denoted $W(f)$, is the set of all points $x \in X$ such that there is an open set $U$ with $x \in U$ such that $U \cap f^{n}(U)$ is empty for all integers $n \neq 0$. A point is nonwandering if it is not wandering.

It is trivial to see that the wandering points form an open subset of $X$ and the nonwandering points a closed subset. Clearly, if $W(f)$ is nonempty, then there can be no invariant arealike probability measure, for the sets $f^{-n}(U)$ must be disjoint sets with the same positive measure, giving $X$ infinite measure. Thus, a nontrivial wandering set puts some restrictions on the types of invariant measures that can exist. However, the wandering set is the easy case to deal with. A few examples on the plane will illustrate some of the main possibilities. A translation is a trivial example of an area-preserving map in which every point is wandering. Rotations are trivial examples of area-preserving maps in which 
wandering points do not exist. The map $f(x, y)=(x / 2, y / 2)$ is an example in which no arealike invariant measure exists, and the wandering set is all points except the origin. Comparing this to the example $f(x, y)=(x / 2,2 y)$, which is area preserving, shows why the wandering set is actually the easy case to deal with. In both cases there are points $x$ such that $f^{n}(x)$ converges to 0 . However, in the latter case, every nonempty open set contains points that go off to infinity in both their forward and backward iterates. This, in fact, is exactly what is needed. To get the more general case, we first need a precise definition of what it means to go to infinity in the more general setting.

Definition. If $X \in \Gamma, x \in X$, and $f \in H(X)$, then $x$ is called an infinity point of $f$ if for every compact subset $K$ of $X$, the set $\left\{n: f^{n}(x) \in K, n\right.$ an integer $\}$ is finite. Note that we include both the forward and backward iterates of $x$ in this definition.

When constructing our invariant measures, it is useful to consider various measures that satisfy certain local properties and then glue them together to get the desired measure. The following easy proposition shows how we do this.

Proposition 1. Let $X$ be a locally compact, separable, metric space, let $f: X \rightarrow X$ be continuous; and suppose that for every nonempty open subset $U$ of $X$, there is an atomless Borel measure $\mu_{U}$ on $X$ that is finite on compact sets and invariant with respect to $f$ such that $\mu_{U}(U)>0$. Then there is an arealike measure $\mu$ on $X$ that is invariant with respect to $X$. If, in addition, the above measures $\mu_{U}$ are all probability measures, then the measure $\mu$ can also be constructed to be a probability measure.

Proof. Since such a space must necessarily be $\sigma$-compact, there is a collection $B_{n}(n=1,2,3, \ldots)$ of compact subsets of $X$ whose union is $X$, such that $B_{n} \subseteq \operatorname{int}\left(B_{n+1}\right)$ for each $n$. Let the sets $U_{n}(n=1,2,3, \ldots)$ form a countable basis of nonempty open subsets of $X$, and for each positive integer $n$, let $\mu_{n}$ be an atomless Borel measure on $X$ that is finite on compact sets, invariant with respect to $f$, such that $\mu_{n}\left(U_{n}\right)>0$. Define $\mu(S)=\sum_{n=1}^{\infty} \mu_{n}(S) /\left(2^{n} \mu_{n}\left(B_{n}\right)\right)$. It is easy to check that $\mu$ (or a scaled multiple) is as desired.

Theorem 1. Let $X \in \Gamma, f \in H(X)$, and suppose $W(f)$ is a dense subset of $X$. Then there is an arealike measure $\mu$ that is invariant with respect to $f$ if and only if every nonempty open subset of $W(f)$ contains an infinity point of $f$. (Note that we could have replaced $W(f)$ by $X$ in the last sentence, but this wording makes the statement of Question 1 easier to give.)

Proof. Suppose there is an arealike measure $\mu$ that is invariant with respect to $f$. Let $\mathrm{U}$ be any nonempty open subset of $W(f)$. Let $B_{n}(n=1,2,3, \ldots)$ be a collection of compact subsets of $X$ whose union is $X$, and such that $B_{n}$ is contained in the interior of $B_{n+1}$. By taking a smaller open set if necessary, we may assume, without loss of generality, that $U \cap f^{n}(U)$ is empty for all integers $n \neq 0$ since $U$ is contained in the wandering set. We may also assume that $\mu(U)<\infty$. For each pair $N, n$ of positive integers, let $D(N, n)=\{x \in$ $U: f^{k}(x) \in B_{n}$ for some $k$ such that $\left.|k|>N\right\}$. Since the sets $f^{n}(U)$ are pairwise disjoint, each $B_{n}$ has finite $\mu$-measure, and $\mu$ is invariant, we have that for each positive integer $n$, there must be a positive integer $N_{n}$ such that $\mu\left(D\left(N_{n}, n\right)\right)>\mu(U)\left(1-1 / 2^{n}\right)$. Thus there is a point $x \in U$ such that $x \in D\left(N_{n}\right)$ for all $n$. It is clear that $x$ must be an infinity point. 
Now, suppose that every nonempty open subset of the wandering set contains an infinity point. Let $B_{n}(n=1,2,3, \ldots)$ be as in the previous paragraph. Let $U$ be any nonempty open set. Let $S$ be the set of all finite sequences of 0 's and 1's. If $s \in S$ is a sequence of length $n$, then we let $s+(0)$ and $s+(1)$ be the sequences of length $n+1$ that are obtained by appending a 0 or 1 (respectively) to the end of the sequence $s$ and define $L(s)$ to be the length of the sequence $s$. For each $s \in S$, we define points $x_{s} \in X$ and open sets $W_{s} \subseteq X$, and for each positive integer $n$ we define a positive integer $N_{n}$ such that the following properties hold.

(1) $x_{s} \in W_{s}$, and $x_{s}$ is an infinity point.

(2) $x_{s+(0)}=x_{s}$.

(3) $\operatorname{cl}\left(W_{e}\right) \subseteq U \cap W(f)$ (where $e$ is the sequence of length zero).

(4) $\operatorname{cl}\left(W_{s+(k)}\right) \subseteq W_{s}, k=0,1$.

(5) $\operatorname{cl}\left(W_{s+(0)}\right)$ and $\operatorname{cl}\left(W_{s+(1)}\right)$ are disjoint compact sets.

(6) If $L(s)=m$, then $f^{j}\left(x_{s}\right) \notin B_{m}$ for all $|j| \geq N_{m}$.

(7) If $L(s) \geq m,|j|<N_{m}$, and $f^{j}\left(X_{s}\right) \notin B_{m}$, then $f^{j}\left(W_{s}\right)$ and $B_{m}$ are disjoint.

(8) If $m$ is the length of $s$, then $W_{s}$ has diameter less than $1 / m$.

The definition proceeds by induction. Suppose $n$ is a positive integer, $x_{s}$ and $W_{s}$ have been defined for all $s$ having length less than $n$, and $N_{m}$ has been defined for all $m<n$. If $s$ is a sequence of length $n-1$, let $x_{s+(0)}=x_{s}$ (so (2) is automatic), and let $x_{s+(1)}$ be an infinity point in $W_{s}$ that is different than $x_{s}$. Such a point exists since $X$ has no isolated points and every open set contains an infinity point. Since there are only finitely many sequences of 0 's and 1's of length $n$ and all of the points $x_{s}$ are infinity points, there is a positive integer $\mathrm{N}$ such that if $s$ has length $n$, then $f^{j}\left(x_{s}\right) \notin B_{n}$ for all $|j| \geq N$. Define $N_{n}$ to be this integer $N$. Since there are only finitely many conditions that need to be satisfied in (7) for $N_{n}$, it is easy to see that the sets $W_{s}$ can be chosen to be small enough so that the remaining conditions hold. Let

$$
C=\bigcap_{n=1}^{\infty} \bigcup_{L(s)=n} W_{s}
$$

or equivalently, $C$ is the closure of the set consisting of all of the points $x_{s}$. Then it easy to check from the above conditions that $C$ is a Cantor set such that $C \cap f^{j}(C)$ is empty for all nonzero $j$ and that for each positive integer $n$, $f^{j}(C) \cap B_{n}$ is empty for all but finitely many $j$. Thus, we can put any atomless probability measure on $C$ (for example, the standard product measure), and use $f$ to induce the appropriate measures on $f^{j}(C)$. We assign measure zero to all sets that are disjoint from all of the sets $f^{j}(C)$, and by Proposition 1, we are done.

If the nonwandering set has interior, then the situation is more complicated. In the original version of this paper, I asked if the hypothesis that $W(f)$ is a dense subset of $X$ could be removed from Theorem 1. Later, C. Simó pointed out a result due to Garrido and himself that shows the answer is no if $X$ is the torus. I also found an easy example on the Cantor Set (Example 1). Since these examples make strong use of properties possessed by the spaces in question, it is not clear that they generalize, and hence suggests the following modification of my original question. 
Question 1. Are there any spaces (for example, the plane) for which the hypothesis that $W(f)$ is a dense subset of $X$ be removed from the above theorem?

If the answer to this question turned out to be yes, then we would have a very nice topological characterization for the existence or nonexistence of arealike invariant measures. If the answer to the question is no, then we can still try to get a more complicated equivalence by adding more conditions. In fact, we will see that this is possible if we restrict ourselves to probability measures. We will also be able to provide a restatement of Question 1 in terms of the existence of a certain type of example.

Definition. Let $X \in \Gamma, f \in H(X)$. If $x \in X$ and $S \subseteq X$, define $P_{n}(x, S)$ to be $N / n$, where $N$ is the number of distinct values of $k, 1 \leq k \leq n$ such that $f^{k}(x) \in S$, i.e., $P_{n}(x, S)$ is the probability that such a $f^{k}(x)$ will be an element of $S$. Given such an $x$ and $S$, we say that $x$ lingers in $S$ iff the limit of the sequence $\left\langle P_{n}(x, S): n=1,2,3, \ldots\right\rangle$ exists and is equal to one. We say that $x$ frequents $S$ iff the above sequence does not have limit zero (i.e., the limit either fails to exist or exists and is nonzero). Note that $x$ lingers in $S$ if and only if $x$ does not frequent the complement of $S$. Of course, the definitions of the terms "lingers" and "frequents" depend on the function $f$, but which function is being used will be clear from context when these terms are used below.

Theorem 2. Let $X \in \Gamma, f \in H(X)$. Then the following are equivalent:

(1) There is an arealike probability measure that is invariant with respect to $f$.

(2) For every nonempty open set $U$ and countable set $C$, there is an $x \in X$ and a compact set $K \subseteq U-C$ such that $x$ frequents $K$.

Proof. (1) $\Rightarrow(2)$ Suppose $\mu$ is an arealike measure on $X$ that is invariant with respect to $f$. Let $U$ be a nonempty open subset of $X$ and let $C$ be a countable subset of $U$. Since $U$ is uncountable (locally compact plus no isolated points implies that every nonempty open set contains a copy of the Cantor set), (2) would be trivially true if $f$ is the identity on $U$, so assume that $f$ is not the identity on $U$. Let $V$ be a nonempty open set whose closure is compact and contained in $U$, and such that $V \cap f(V)$ is empty. Let $W$ be an open set such that $C \subseteq W$ and $\mu(W)<\mu(V)$. Such a $W$ exists since $C$ has measure zero. Let $K=\operatorname{cl}(V)-W$. We will now show that some element of $X$ frequents $K$. Note that $\mu(K)>0$. Let $N$ be a positive even integer such that $N \mu(K)>1$. Fix an integer $k>0$. For $1 \leq n \leq k N$, let $Z_{n}$ be the set of all points $x \in X$ such that there are at least $n$ different values of $i$, $1 \leq i \leq k N$, such that $f^{i}(x) \in K$. Let $Y_{k}=Z_{k / 2}$. Then

$$
\sum_{n=1}^{k N} \mu\left(Z_{n}\right)=\sum_{n=1}^{k N} \mu\left(f^{-n}(K)\right)=k N \mu(K)>k
$$

Note that for all $i, f^{i}(K)$ and $f^{i+1}(K)$ are disjoint, so $Z_{n}$ is empty for 
$n>(k N / 2)$. Note also that $Z_{n+1} \subseteq Z_{n}$. Thus

$$
\begin{aligned}
\sum_{n=1}^{k N} \mu\left(Z_{n}\right) & =\sum_{n=1}^{k N / 2} \mu\left(Z_{n}\right) \leq \sum_{n=1}^{k / 2} \mu\left(Z_{1}\right)+\sum_{n=k / 2+1}^{k N / 2} \mu\left(Z_{k / 2}\right) \\
& \leq k / 2+k(N-1) \mu\left(Z_{k / 2}\right) / 2 .
\end{aligned}
$$

Thus $k / 2+k(N-1) \mu\left(Z_{k / 2}\right) / 2>k$ and $\mu\left(Z_{k / 2}\right)=\mu\left(Y_{k}\right)>1 /(N-1)$. Note that if $x \in Y_{k}$ then $P_{k}(x, K)>1 /(N-1)$. Let $x$ be such that $x \in Y_{k}$ for infinitely many $k$. Such an $x$ must exist since the sets $Y_{k}$ all have measure at least $1 /(N-1)$. Then $x$ frequents $K$, and we are done.

(2) $\Rightarrow(1)$. This direction is much more complicated and requires a closer look at how invariant measures are constructed. The proof given here is mainly topological and looks somewhat different from the standard construction of invariant measures, although the underlying ideas are basically the same. It seems likely that a different proof of this result could be given by the more standard route via the Riesz Representation Theorem, but the details for such a possible alternate proof have not been worked out.

We remind the reader of the basic outlines of the standard construction of a countably additive measure from a finitely additive measure, using open covers (see [OU], for example). Let $m$ be a countably additive measure on the Borel subsets of $X$, and for each $B \subseteq X$ the outer measure $\mu^{*}(B)$ is defined to be the infimum of all sums $\sum_{U \in C} m(U)$, where $C$ ranges over all open covers of $B$. If we let $\mu$ be the restriction of $\mu^{*}$ to the Borel sets $B$, then $\mu$ is a countably additive Borel measure on $X$ and will be invariant with respect to the function $f$ if $m$ was. While $\mu$ may give different measure to a set than $m$, we do have the following easy facts:

(a) If $U$ is open, then $\mu(U) \leq m(U)$.

(b) If $B$ is compact, then $\mu(U) \geq m(U)$.

Now, suppose (2) holds. Let $U$ be an arbitrary nonempty open subset of $X$. By Proposition 1, it suffices to prove that there is an atomless Borel probability measure $\mu$ on $X$ such that $\mu(U)>0$ and $\mu$ is preserved by $f$. We must divide into two cases.

Case I. $U$ contains uncountably many periodic points. Then there is a positive integer $n$ such that $f^{n}(x)=x$ for uncountably many $x \in U$, and we may assume $\mathrm{n}$ is the least such integer. Since $\left\{x \in X: f^{n}(x)=x\right\}$ is a closed subset of $X$, it is easy to see (by the leastness of $n$ ) that there must be a Cantor set $C \subseteq U$ such that the sets $C, f(C), f^{2}(C), \ldots, f^{n-1}(C)$ are pairwise disjoint and $f^{n}(x)=x$ for all $x \in C$. There is an atomless Borel measure $\mu$ on $C$ such that $\mu(C)=1 / n$, and this induces obvious measures on $f(C), f^{2}(C), \ldots, f^{n-1}(C)$. Letting $\mu$ be zero outside the sets $C, f(C), f^{2}(C), \ldots, f^{n-1}(C)$ gives us our desired measure.

Case II. $U$ contains only countably many periodic points. Then by (2), there is a compact set $K \subseteq U$ containing no periodic points and a point $x \in X$ that frequents $K$. Since $x$ frequents $K$, there is a positive number $\varepsilon$ and an infinite set $J$ of positive integers such that for each $n \in J, P_{n}(x, K) \geq \varepsilon$. Let $\beta N$ be the Stone-Čech compactification of the set $N$ of positive integers, and let $p$ be a limit point of $J$ in $\beta N$. We remind the reader that one basic property of the Stone-Čech compactification $N$ is that any bounded continuous real-valued function on $N$ can be extended uniquely to a continuous real-valued 
function on $\beta N$. Thus given any subset $S$ of $X$, we can define the real-valued function $g_{S}$ on $N$ by $g_{S}(n)=P_{n}(x, S)$, and each of these function extends to a continuous function on $\beta N$ (which we shall still call $g_{S}$ ). We now define our finitely additive measure $m$ by $m(S)=g_{S}(p)$, for each subset $S$ of $X$. If $S$ and $T$ are disjoint subsets of $X$, then it is easy to see from the definition that $P_{n}(x, S)+P_{n}(x, T)=P_{n}(x, S \cup T)$ for all $n$, and thus, by continuity of (the extension of) $g_{S}, g_{T}$, and $g_{S \cup T}$, that $m(S \cup T)=m(S)+m(T)$. Also, from the definition of $P_{n}(x, S)$, it obvious that $\left|P_{n}(x, S)-P_{n}\left(x, f^{-1}(S)\right)\right| \leq 2 / n$ (actually, it is no more than $1 / n$, as can be seen on closer examination, but we do not need that much precision). Thus, we again get by the continuity of $g_{S}$ that $m(S)=m\left(f^{-1}(S)\right)$. Also, since $g_{K}(n) \geq \varepsilon$ for all $n \in J$ and $p$ is a limit point of $J$, it follows that $m(K) \geq \varepsilon$. Thus, if we let $\mu$ be the countably additive measure generated by $m$, then $\mu$ is invariant with respect to $f$ and $\mu(K) \geq m(K)$ since $K$ is compact. Let $C$ be the set of all periodic points of $f$. We define a new measure by removing any part of the measure $\mu$ that gives nontrivial measure to any periodic point, i.e., we define the measure $\nu$ on $X$ by $\nu(S)=\mu(S-C)$. Then, since $K-C=K$, it follows that $\nu(K)>0$, and therefore $\nu(U)>0$. Since $X$ is open, $\nu(X) \leq \mu(X) \leq m(X)=1$, so $\nu$ can be scaled to a probability measure. Since $\nu(X)$ is finite, any point having nonzero measure would have to be periodic, but we made sure that $\nu(C)=0$, so every point has measure zero, and we are done.

Theorems 1 and 2 can be combined to get a sufficient (but perhaps not necessary) condition for the existence of an arealike invariant measure for a homeomorphism of some $X \in \Gamma$. Going back again to Proposition 1 , if $U$ is a nonempty open subset of $X$, then there is some nonempty open subset of $U$ in which every point is either wandering (in which case one appeals to Theorem 1) or nonwandering (in which Theorem 2 is of use). However, if the nonwandering set fails to be compact, then it is not clear that we get a necessary condition, so the most general case of getting a reasonable necessary and sufficient condition for all elements of $\Gamma$ still has a small gap. The nicest outcome would be if Question 1 has a positive answer for the plane. However, even for those spaces for which the answer to Question 1 is no, we would like to know what a typical counterexample would look like. This motivates the following question.

Question 2. Let $X \in \Gamma$. Does there exist a homeomorphism $f$ on $X$ having only countably many (perhaps only one) periodic points, no wandering points, and having the additional property that if $U$ is any open set containing all of the periodic points, then every point of $X$ lingers in $U$ ?

It is easy to see that there is a relationship between Questions 1 and 2. For a given space $X$, an answer of yes to Question 2 easily gives an answer of no to Question 1 (using Theorem 2). In the other direction, an answer of no to Question 1 for the space $X$ means that for some open subset $U$ of $X$, either $U$ or the one-point compactification of $U$ answers Question 2 in the positive.

Example 1. Let $X$ be the Cantor Set, viewed as the set of all biinfinite sequences of 0 's and 1's. Let $S$ be the set of all such sequences having the property that for each positive integer $n$, every consecutive sequence of $n^{2}$ entries has no more than $n$ 1's. Let $f$ be the usual shift homeomorphism. 
It is easily seen that both Example 1 and the Garrido-Simó example mentioned above give positive answers to Question 2, and therefore negative answers to Question 1.

The space that motivated this paper is the plane, and the study of areapreserving homeomorphisms of the plane has generated considerable interest in recent years. It is therefore of particular interest whether or not Question 1 is true when $X$ is the plane. In [BS], for example, the Brouwer Lemma played a crucial role, and this result is not true for the torus, the only known 2-manifold giving a negative answer to Question 1. Thus, it may be that the Brouwer Lemma, or a similar result, will eventually be of use in proving a stronger version of these results when $X$ is the plane.

\section{REFERENCES}

[BS] Stewart Baldwin and Edward E. Slaminka, On measures preserved by Brouwer homeomorphisms, Topology Proc. 14 (1989), 1-6.

[GS] L. Garrido and C. Simó, Some ideas about strange attractors, Lecture Notes in Phys., vol. 179, Springer-Verlag, 1982, pp. 1-28.

[KB] N. Kryloff and N. Bogoliouboff, La théorie générale de la mesure dans son application à l'étude des systems dynamique de la mécanique non linéaire, Ann. of Math. (2) 38 (1937), 65-113.

[OU] J. C. Oxtoby and S. M. Ulam, Measure preserving homeomorphisms and metrical transitivity, Ann. of Math. (2) 42 (1941), 874-920.

[W] Peter Walters, An introduction to ergodic theory, Springer-Verlag, New York, Heidelberg and Berlin, 1982.

Department of Foundations, Analysis, and Topology, Auburn University, Auburn, Alabama 36849-5310 